\title{
Síndrome Inflamatória Multissistêmica Pediátrica (SIM-P): Uma análise epidemiológica brasileira
}

\author{
Multisystem Inflammatory Syndrome in Children (MIS-C): A Brazilian epidemiological analysis \\ Síndrome Inflamatorio Multisistémico Pediátrico (SIM-P): Un análisis epidemiológico brasileño
}

Murilo Luiz Louzada Brandão ORCID: https://orcid.org/0000-0003-1609-5158 Centro Universitário Atenas, Brasil

E-mail: muriloluizlbrandao@gmail.com

Fernanda Odete Souza Rodrigues ORCID: https://orcid.org/0000-0002-7340-4351 Universidade de Itaúna, Brasil

E-mail: nandasouzarodrigues@hotmail.com Lidiane Bernardo Gomes ORCID: https://orcid.org/0000-0002-7416-7191 Centro Universitário Doutor Leão Sampaio, Brasil

E-mail: lidianegeo.lb@gmail.com

Edson Luiz Brandão Netto

ORCID: https://orcid.org/0000-0003-2541-881X Centro Universitário IMEPAC Araguari, Brasil E-mail: elbnedsonnetto@gmail.com

Brenda Nayara Gontijo de Moura ORCID: https://orcid.org/0000-0003-2228-289X Universidade de Itaúna, Brasil

E-mail: brendagontijodemoura@gmail.com

Bianca Cardoso Lopes

ORCID: https://orcid.org/0000-0001-8619-1749

Universidade Federal de Ouro Preto, Brasil E-mail: biancalopesc50@gmail.com

Rafael Vinícius de Assis Menezes ORCID: https://orcid.org/0000-0003-1813-9919

Universidade Federal de Ouro Preto, Brasil

E-mail: rafael.menezes@aluno.ufop.edu.br

Leonardo Alexandre do Amaral ORCID: https://orcid.org/0000-0002-7198-636X

Universidade Federal de Ouro Preto, Brasil E-mail: leoale01@gmail.com

Brenio Felipe Batista Pereira ORCID: https://orcid.org/0000-0002-8246-1200

Universidade Federal de Ouro Preto, Brasil E-mail: brenio.fbp@gmail.com

Thaís Fernandes Campos

ORCID: https://orcid.org/0000-0001-8162-9393

Centro Universitário Funorte, Brasil

E-mail: thaiscampos26@gmail.com

Isadora Porto de Aquino

ORCID: https://orcid.org/0000-0001-6800-3396 Universidade de Itaúna, Brasil

E-mail: isadoraportoaquino@gmail.com

Déborah Luiza Vilela de Oliveira

ORCID: https://orcid.org/0000-0002-0150-9218

Universidade Federal de Ouro Preto, Brasil

E-mail: deborah_luiza97@hotmail.com

\begin{abstract}
Resumo
O presente artigo objetivou analisar o contexto epidemiológico brasileiro da síndrome inflamatória multissistêmica pediátrica (SIM-P) temporalmente associada à Covid-19. Para tanto, realizou-se um estudo estatístico e descritivo, pautado pela interpretação de dados coletados nas plataformas das Secretarias de Saúde das 27 unidades federativas do país e que abrangeram o período entre o primeiro caso confirmado da síndrome, em 2020, até junho de 2021 . Os
\end{abstract}


parâmetros investigados versaram sobre o número total de casos e de óbitos, bem como à distribuição entre estados, sexos e faixas etárias. Foram registrados 1010 diagnósticos e 65 óbitos desencadeados pela síndrome, indicando uma taxa de letalidade de 6,4\%. 55,4\% dos casos foram descritos no sexo masculino, enquanto os óbitos, por sua vez, foram mais comuns em meninas, representando, aproximadamente, $76 \%$ das mortes verificadas. A faixa etária de maior acometimento da SIM-P foi entre 0 e 4 anos de idade. A maior parte dos casos e das mortes pela síndrome ocorreram na região sudeste, sobretudo no estado de São Paulo, que comportou 19,1\% do total de diagnósticos e $18,4 \%$ da totalidade de falecimentos do Brasil. A SIM-P representa condição grave associada à infecção pelo SARSCoV-2 e, por isso, exige cautela por parte de crianças, adolescentes e de seus familiares quanto à adequada adoção de medidas preventivas recomendadas para a Covid-19. Ainda que se trate de tema recente, os números já notificados no país são suficientes para que pediatras e generalistas obtenham o devido conhecimento médico para o diagnóstico precoce desta síndrome, permitindo a redução dos desfechos negativos e seu melhor prognóstico.

Palavras-chave: Síndrome inflamatória multissistêmica; Covid-19; Epidemiologia; Pediatria; Brasil.

\begin{abstract}
This article aimed to analyze the Brazilian epidemiological context of pediatric multisystemic inflammatory syndrome (MIS-C) temporally associated with Covid-19. Therefore, a statistical and descriptive study was carried out, guided by the interpretation of data collected on the platforms of the Health Departments of the 27 federative units in the country and covering the period between the first confirmed case of the syndrome, in 2020, until june 2021. The parameters investigated were about the total number of cases and deaths, as well as the distribution between states, sexes and age groups. There were 1010 diagnoses and 65 deaths triggered by the syndrome, indicating a fatality rate of $6.4 \% .55 .4 \%$ of the cases were described in males, while deaths, in turn, were more common in girls, representing approximately $76 \%$ of the verified deaths. The age group most affected by SIM-P was between 0 and 4 years of age. Most cases and deaths caused by the syndrome occurred in the Southeast region, especially in the state of São Paulo, which accounted for $19.1 \%$ of all diagnoses and $18.4 \%$ of all deaths in Brazil. SIM-P represents a serious condition associated with SARS-CoV-2 infection and, therefore, requires caution on the part of children, adolescents and their families regarding the proper adoption of preventive measures recommended for Covid-19. Although this is a recent topic, the numbers already reported in the country are sufficient for pediatricians and general practitioners to obtain the necessary medical knowledge for the early diagnosis of this syndrome, allowing for the reduction of negative outcomes and a better prognosis.
\end{abstract}

Keywords: Multisystemic inflammatory syndrome; Covid-19; Epidemiology; Pediatrics; Brazil.

\title{
Resumen
}

Este artículo tenía como objetivo analizar el contexto epidemiológico brasileño del síndrome inflamatorio multisistémico pediátrico (SIM-P) asociado temporalmente a Covid-19. Por ello, se realizó un estudio estadístico y descriptivo, guiado por la interpretación de los datos recolectados en las plataformas de los Departamentos de Salud de las 27 unidades federativas del país y cubriendo el período comprendido entre el primer caso confirmado del síndrome, en 2020, hasta Junio de 2021 Los parámetros investigados fueron sobre el número total de casos y muertes, así como la distribución entre estados, sexos y grupos de edad. Hubo 1010 diagnósticos y 65 muertes provocadas por el síndrome, lo que indica una tasa de letalidad del 6,4\%. 55,4\% de los casos se describieron en hombres, mientras que las muertes, a su vez, fueron más frecuentes en las niñas, representando aproximadamente el $76 \%$ de las muertes. El grupo de edad más afectado por SIM-P fue el de 0 a 4 años. La mayoría de los casos y muertes causadas por el síndrome ocurrieron en la región sureste, especialmente en el estado de São Paulo, que representó el 19,1\% de todos los diagnósticos y el 18,4\% de todas las muertes en Brasil. SIM-P representa una condición grave asociada a la infección por SARS-CoV-2 y, por lo tanto, requiere precaución por parte de los niños, adolescentes y sus familias con respecto a la adecuada adopción de las medidas preventivas recomendadas para Covid-19. Si bien este es un tema reciente, las cifras ya reportadas en el país son suficientes para que los pediatras y médicos generales obtengan los conocimientos médicos necesarios para el diagnóstico precoz de este síndrome, permitiendo la reducción de los resultados negativos y un mejor pronóstico.

Palabras clave: Síndrome inflamatorio multisistémico; Covid-19; Epidemiología; Pediatría; Brasil. 


\section{Introdução}

Em março de 2020, a Organização Mundial da Saúde (OMS) oficializou o estado de pandemia, decretado após a disseminação global do vírus SARS-CoV-2. O surto inicial teve origem na China, na cidade de Wuhan, e desencadeou, mundialmente, preocupações que atingem diversas esferas do cotidiano humano, com repercussões significativas sobre a saúde e as dinâmicas financeiras individuais e nacionais. No Brasil, o primeiro caso confirmado data de 26 de fevereiro de 2020 e, até a segunda semana de julho de 2021, mais de 19,1 milhões de diagnósticos foram atestados e, aproximadamente, 533 mil óbitos contabilizados (Organização Mundial da Saúde, 2021; Ministério da Saúde, 2021).

O histórico do coronavírus, tanto as formas antigas quanto a atual com suas crescentes variantes, demonstra notável acometimento do sistema respiratório, com transmissão interpessoal relacionada à inalação de secreções e ao contato direto. Desse modo, o quadro clínico clássico determinado pelo patógeno contempla, principalmente, manifestações como febre, tosse, dispneia, mialgia, fadiga e anosmia. O prognóstico da doença tende a ser pior em pessoas com comorbidades prévias e em determinados grupos etários, podendo ocorrer prejuízo no diagnóstico precoce devido à apresentação assintomática ou levemente sintomática da infecção, a exemplo do que ocorre na maioria das crianças com Covid-19 (Sáfadi, 2020).

De acordo com o Centro de Controle e Prevenção de Doenças (CDC), em junho de 2021, crianças e adolescentes perfizeram cerca de $13 \%$ da população comprovadamente infectada pelo SARS-CoV-2 nos Estados Unidos. Ainda que o número indique menor frequência da doença entre pessoas com idade entre 0 e 19 anos, possivelmente justificada pela reduzida quantidade de receptores da enzima conversora de angiotensina II ou pela maior ativação da resposta imune inata, casos com evolução grave têm sido registrados e incluem desfechos como obstrução intestinal, falência múltipla de órgãos e uma reação inflamatória multissistêmica (SIM), similar à doença de Kawasaki (DK) e à síndrome do choque tóxico (Centro de Controle e Prevenção de Doenças, 2021; Silva et al., 2020).

Sobre a SIM, os primeiros relatos foram registrados no Reino Unido, em abril de 2020. A condição tem sido descrita como rara em todo o mundo, com recuperação completa na maioria das vezes, mas com algumas evidências de prognóstico reservado em razão do comprometimento de órgãos e sistemas variados. Diante de sua potencial gravidade e da constatação sobre a expressiva correlação com a infecção vigente ou prévia pelo novo coronavírus, o CDC emitiu, em maio de 2020, comunicado que consolidou o quadro clínico como síndrome inflamatória multissistêmica pediátrica (SIM-P) e que sinalizou alerta mundial para possíveis novas repercussões causadas pelo SARS-CoV-2 (Feldstein et al., 2020).

Em resposta, em julho de 2020, o Ministério da Saúde brasileiro (MS) formalizou a orientação sobre a notificação compulsória da SIM-P no país, permitindo rastreio efetivo da afecção. No entanto, embora mais de um ano tenha se passado desde o primeiro caso confirmado no mundo, o mecanismo pelo qual o estado hiperinflamatório multissistêmico é desencadeado persiste indefinido, ao contrário do que ocorre na Covid-19, cujas teorias fisiopatológicas são razoavelmente bem estabelecidas. Uma vez que o estudo aprofundado sobre a síndrome é recente, posto o atual crescimento progressivo desses diagnósticos, uma série de questionamentos permanece sem conclusões, representando preocupações para generalistas, pediatras e autoridades de saúde (Martins et al., 2020; Fontes et al., 2021).

Quanto às apreensões geradas pela SIM-P, quando analisada a atual conjuntura brasileira, ressalta-se a possibilidade de volta às aulas presenciais, bem como as pressões por autorizações ao retorno de grandes eventos, sendo, estes, comumente frequentados por jovens. Todavia, tendo em vista a situação da vacinação no país, que indica a imunização completa, até o momento, de apenas $18,1 \%$ da população vacinável, ambas as situações expõem pessoas suscetíveis à infecção pelo SARSCoV-2 (Ministério da Saúde, 2021). Nessas circunstâncias, o aumento do risco de contágio pode impactar na elevação no número casos de Covid-19 pediátrica no país e, consequentemente, oportunizar o desenvolvimento da SIM-P (Godfred-Cato et al., 2020). 
Frente a esse cenário, o presente artigo visou analisar o contexto epidemiológico brasileiro da SIM-P pós-Covid, sintomatologia grave, importante e de conhecimento e reconhecimento ainda incipientes. Por esse motivo, também integra os objetivos desta pesquisa o fomento à elaboração de novos estudos científicos sobre essa temática, de modo a permitir amplo entendimento sobre os aspectos que essa afecção abrange.

\section{Metodologia}

Trata-se de estudo descritivo e de cunho epidemiológico, embasado pela análise de dados referentes aos casos de SIM-P pós-Covid no Brasil, coletados nas plataformas digitais de cada uma das 27 Secretarias de Saúde pertencentes às unidades da federação e disponibilizados por Boletins Epidemiológicos do Ministério da Saúde. A seleção das informações foi realizada ao final de junho de 2021 e os registros retrataram o período que se estendeu desde o primeiro caso confirmado em cada estado, ainda em 2020, até junho de 2021, último mês em que os números foram avaliados.

Sobre as informações encontradas, objetivou-se investigar e interpretar aquelas relacionadas ao número total de casos e de óbitos desencadeados pela síndrome, bem como as que se referiram à distribuição da afecção entre faixas etárias, sexos e estados brasileiros. Após ordenação sequencial dos dados, os resultados conclusivos foram alocados sob a forma de tabela e as análises concretizadas a partir de suas verificações foram relatadas de formas descritiva e estatística no decorrer desta pesquisa.

Integra, ainda, este artigo, uma revisão bibliográfica de produções científicas publicadas entre os anos de 2018 e 2021 e indexadas nas bases de dados Scientific Electronic Library Online (SciELO), National Library of Medicine (PubMED) e Google Acadêmico. Para tanto, os descritores utilizados na busca foram "síndrome de resposta inflamatória sistêmica", "Covid-19", "pediatria", "epidemiologia" e "Brasil" e seus respectivos correspondentes em inglês e espanhol. Apenas textos originais foram selecionados, enquanto aqueles que se apresentaram em duplicidade ou que não contemplaram, diretamente, a temática deste estudo, foram descartados

\section{Resultados}

O período analisado nesta pesquisa abrangeu desde o início da notificação compulsória da SIM-P no Brasil até o dia 22 de junho de 2021, data da coleta de dados nas plataformas das Secretarias de Saúde dos 26 estados e do Distrito Federal. Nesse intervalo, foram confirmados, em todo o país, 1010 diagnósticos e 65 óbitos desencadeados pela síndrome, representando uma taxa de letalidade de, aproximadamente, 6,4\%. Abaixo, a tabela indica a distribuição, por faixa etária e sexo, de casos e óbitos nas unidades da federação (Tabela 1).

Tabela 1 - Distribuição de casos de SIM-P nas unidades da federação brasileira conforme evolução, sexo e faixa etária.

Distribuição por faixa etária e sexo

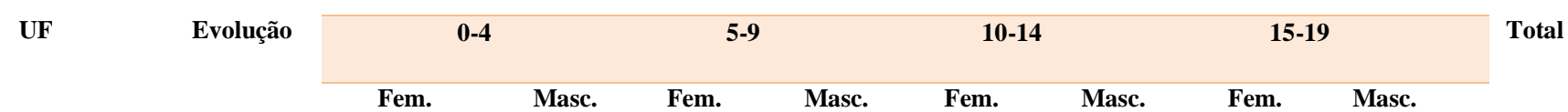

\begin{tabular}{cccccccccccc}
\hline Acre & Casos & 1 & 2 & 0 & 0 & 1 & 0 & 0 & 0 \\
& Óbitos & 0 & 1 & 0 & 0 & 0 & 0 & 0 & 0 \\
\hline Alagoas & Casos & 8 & 13 & 7 & 6 & 2 & 8 & 0 & 0 \\
\hline
\end{tabular}


Research, Society and Development, v. 10, n. 9, e44710918154, 2021

(CC BY 4.0) | ISSN 2525-3409 | DOI: http://dx.doi.org/10.33448/rsd-v10i9.18154

\begin{tabular}{|c|c|c|c|c|c|c|c|c|c|c|}
\hline & Óbitos & 0 & 0 & 0 & 0 & 0 & 1 & 0 & 0 & 1 \\
\hline \multirow[t]{2}{*}{ Amapá } & Casos & 0 & 0 & 0 & 0 & 0 & 0 & 0 & 0 & 0 \\
\hline & Óbitos & 0 & 0 & 0 & 0 & 0 & 0 & 0 & 0 & 0 \\
\hline \multirow[t]{2}{*}{ Amazonas } & Casos & 25 & 0 & 0 & 0 & 0 & 0 & 0 & 0 & 0 \\
\hline & Óbitos & 5 & 0 & 0 & 0 & 0 & 0 & 0 & 0 & 0 \\
\hline \multirow[t]{2}{*}{ Bahia } & Casos & 16 & 20 & 18 & 7 & 0 & 14 & 1 & 3 & 79 \\
\hline & Óbitos & 1 & 2 & 1 & 1 & 0 & 0 & 0 & 0 & 5 \\
\hline \multirow[t]{2}{*}{ Ceará } & Casos & 15 & 10 & 7 & 11 & 12 & 7 & 0 & 2 & 64 \\
\hline & Óbitos & 0 & 0 & 0 & 0 & 2 & 0 & 0 & 0 & 2 \\
\hline \multirow[t]{2}{*}{ Distrito Federal } & Casos & 15 & 9 & 7 & 13 & 7 & 10 & 1 & 1 & 63 \\
\hline & Óbitos & 0 & 0 & 0 & 0 & 0 & 0 & 1 & 0 & 1 \\
\hline \multirow[t]{2}{*}{ Espírito Santo } & Casos & 5 & 4 & 7 & 2 & 2 & 2 & 0 & 0 & 22 \\
\hline & Óbitos & 0 & 0 & 1 & 0 & 0 & 0 & 0 & 0 & 1 \\
\hline \multirow[t]{2}{*}{ Goiás } & Casos & 5 & 4 & 5 & 7 & 1 & 3 & 0 & 0 & 25 \\
\hline & Óbitos & 1 & 1 & 0 & 0 & 0 & 0 & 0 & 0 & 2 \\
\hline \multirow[t]{2}{*}{ Maranhão } & Casos & 1 & 5 & 1 & 3 & 0 & 3 & 0 & 0 & 13 \\
\hline & Óbitos & 1 & 1 & 0 & 0 & 0 & 0 & 0 & 0 & 2 \\
\hline \multirow[t]{2}{*}{ Minas Gerais } & Casos & 17 & 42 & 17 & 26 & 3 & 6 & 0 & 0 & 111 \\
\hline & Óbitos & 1 & 1 & 0 & 1 & 0 & 0 & 0 & 0 & 3 \\
\hline \multirow[t]{2}{*}{ Mato Grosso } & Casos & 2 & 1 & 0 & 3 & 1 & 1 & 0 & 1 & 9 \\
\hline & Óbitos & 0 & 0 & 0 & 0 & 0 & 0 & 0 & 0 & 0 \\
\hline \multirow[t]{2}{*}{ Mato Grosso do Sul } & Casos & 0 & 0 & 0 & 0 & 0 & 0 & 1 & 0 & 1 \\
\hline & Óbitos & 0 & 0 & 0 & 0 & 0 & 0 & 1 & 0 & 1 \\
\hline \multirow[t]{2}{*}{ Pará } & Casos & 16 & 21 & 1 & 11 & 5 & 6 & 0 & 0 & 60 \\
\hline & Óbitos & 5 & 3 & 0 & 1 & 1 & 0 & 0 & 0 & 10 \\
\hline \multirow[t]{2}{*}{ Paraíba } & Casos & 4 & 2 & 1 & 3 & 1 & 0 & 0 & 0 & 11 \\
\hline & Óbitos & 2 & 1 & 0 & 0 & 0 & 0 & 0 & 0 & 3 \\
\hline \multirow[t]{2}{*}{ Paraná } & Casos & 6 & 8 & 6 & 8 & 4 & 3 & 1 & 1 & 37 \\
\hline & Óbitos & 1 & 0 & 0 & 1 & 1 & 0 & 1 & 0 & 4 \\
\hline \multirow[t]{2}{*}{ Pernambuco } & Casos & 5 & 6 & 6 & 6 & 2 & 5 & 0 & 0 & 30 \\
\hline & Óbitos & 1 & 0 & 0 & 0 & 1 & 0 & 0 & 0 & 2 \\
\hline \multirow[t]{2}{*}{ Piauí } & Casos & 1 & 4 & 1 & 1 & 1 & 1 & 0 & 0 & 9 \\
\hline & Óbitos & 0 & 1 & 0 & 0 & 0 & 0 & 0 & 0 & 1 \\
\hline
\end{tabular}




\begin{tabular}{|c|c|c|c|c|c|c|c|c|c|c|}
\hline Rio de Janeiro & Casos & 19 & 21 & 12 & 9 & 5 & 7 & 2 & 1 & 76 \\
\hline & Óbitos & 1 & 4 & 1 & 0 & 0 & 0 & 1 & 0 & 7 \\
\hline \multirow{2}{*}{$\begin{array}{c}\text { Rio Grande do } \\
\text { Norte }\end{array}$} & Casos & 2 & 3 & 1 & 3 & 0 & 3 & 0 & 2 & 14 \\
\hline & Óbitos & 0 & 0 & 0 & 0 & 0 & 0 & 0 & 0 & 0 \\
\hline \multirow[t]{2}{*}{ Rio Grande do Sul } & Casos & 8 & 12 & 10 & 17 & 6 & 7 & 0 & 0 & 60 \\
\hline & Óbitos & 0 & 0 & 0 & 1 & 0 & 0 & 0 & 0 & 1 \\
\hline \multirow[t]{2}{*}{ Rondônia } & Casos & 0 & 0 & 0 & 1 & 0 & 1 & 0 & 0 & 2 \\
\hline & Óbitos & 0 & 0 & 0 & 0 & 0 & 0 & 0 & 0 & 0 \\
\hline \multirow[t]{2}{*}{ Roraima } & Casos & 0 & 0 & 0 & 0 & 0 & 0 & 0 & 0 & 0 \\
\hline & Óbitos & 0 & 0 & 0 & 0 & 0 & 0 & 0 & 0 & 0 \\
\hline \multirow[t]{2}{*}{ Santa Catarina } & Casos & 8 & 13 & 6 & 8 & 4 & 7 & 2 & 0 & 48 \\
\hline & Óbitos & 1 & 0 & 0 & 0 & 0 & 0 & 0 & 0 & 1 \\
\hline \multirow[t]{2}{*}{ São Paulo } & Casos & 28 & 48 & 36 & 35 & 15 & 24 & 6 & 1 & 193 \\
\hline & Óbitos & 0 & 3 & 2 & 1 & 0 & 2 & 4 & 0 & 12 \\
\hline \multirow[t]{2}{*}{ Sergipe } & Casos & 2 & 1 & 1 & 0 & 3 & 0 & 0 & 0 & 7 \\
\hline & Óbitos & 0 & 0 & 0 & 0 & 0 & 0 & 0 & 0 & 0 \\
\hline \multirow[t]{3}{*}{ Tocantins } & Casos & 0 & 1 & 1 & 0 & 1 & 0 & 0 & 0 & 3 \\
\hline & Óbitos & 0 & 0 & 0 & 0 & 0 & 0 & 0 & 0 & 0 \\
\hline & Casos & 209 & 250 & 151 & 180 & 76 & 118 & 14 & 12 & 1010 \\
\hline BRASIL & Óbitos & 19 & 18 & 5 & 6 & 6 & 3 & 8 & $\mathbf{0}$ & 65 \\
\hline
\end{tabular}

Fonte: Adaptado de Ministério da Saúde (2021). Fem.: feminino; Masc.: masculino.

Sobre a alocação epidemiológica e regional da síndrome, observou-se maior concentração da SIM-P na região sudeste, com o registro de 342 casos e 24 mortes, contemplando 33,8\% e 36,9\% dos parâmetros nacionais correspondentes. Da totalidade de diagnósticos de SIM-P e de falecimentos do sudeste, 56,4\% e 50,0\%, respectivamente, foram identificados apenas no estado de São Paulo. Com relação ao contexto nacional, este mesmo estado respondeu por 19,1\% de todos os casos confirmados no período e por 18,4\% dos óbitos causados pela síndrome no Brasil.

Ainda sobre este aspecto e, em contrapartida, diagnósticos de SIM-P após infecção pela Covid-19 foram infrequentes no norte do país e em alguns estados do centro-oeste, destacando-se, entre eles, Amapá, Acre, Mato Grosso do Sul, Rondônia e Roraima, cujas evidências absolutas apontaram para $0,4,1,2$ e 0 casos, nesta ordem. À avaliação do número de eventos fatais por SIM-P no país, apenas 6 das 27 unidades da federação permaneceram sem óbitos, sendo elas Amapá, Mato Grosso, Rondônia, Roraima, Sergipe e Tocantins.

A SIM-P foi mais notificada, na maioria das faixas etárias, no sexo masculino, exceto entre jovens de 15 a 19 anos. Ao todo, 55,4\% dos casos incidiram em meninos, enquanto 44,6\% ocorreram em meninas. No que diz respeito ao número de mortes, todavia, mais ocorrências foram identificadas em meninas $(75,9 \%)$, principalmente a partir dos 9 anos de idade. De modo geral, a maior parte dos estados brasileiros apresentou perfil semelhante ao supracitado, com diferenças e 
particularidades relatadas no Ceará, Espírito Santo, Sergipe, Tocantins e na Paraíba, que obtiveram maior incidência da síndrome em meninas, e nos estados do Amazonas e Mato Grosso do Sul que apresentaram 100\% dos diagnósticos confirmados em crianças e adolescentes do sexo feminino.

Quanto à disposição dos casos e de mortes pela síndrome, de acordo com as faixas etárias, a de maior prevalência da doença, ao se observar o cenário nacional, foi aquela entre 0 a 4 anos, representando 45,4\% do total de diagnósticos notificados e $60,6 \%$ da totalidade de óbitos, respectivamente. No entanto, houve heterogeneidade nas unidades da federação, com estados como Espírito Santo, Mato Grosso e Paraná apresentando incidências semelhantes nas faixas etárias de 0 a 4 e de 5 a 9 anos. Distribuição trimodal e com acometimento de indivíduos com idades superiores foi observada em Tocantins, que confirmou igual número de casos entre 0 e 4,5 a 9 e 10 a 14 anos de idade, sendo a primeira e a última faixas também referidas pelo estado de Sergipe.

\section{Discussão}

A Sociedade Brasileira de Pediatria informou em sua nota técnica sobre a epidemiologia da Covid-19, divulgada em março deste ano, uma sensível redução no percentual de hospitalizações, óbitos e na taxa de letalidade, na população infantojuvenil, entre 2020 e 2021. Em contrapartida, análises mais recentes têm mostrado que os números brasileiros sobre esses parâmetros ainda são alarmantes, se comparados com os de outros países. Confirmam essa constatação os dados disponibilizados pelo MS que indicaram que a mortalidade entre crianças menores de 9 anos, infectadas pelo SARS-CoV-2, foi, aproximadamente, 17 vezes maior do que a registrada no Reino Unido, por exemplo (Ministério da Saúde, 2021; Passarinho \& Barrucho, 2021).

De modo semelhante à população geral, pacientes pediátricos, quando não assintomáticos ou levemente sintomáticos, tendem a ter apresentação clínica com maiores alterações respiratórias, destacando-se, entre elas, coriza, obstrução nasal, laringite e faringite, tosse e odinofagia. $\mathrm{Na}$ medida em que a infecção se torna prolongada, acometimentos do trato gastrointestinal e manifestações neurológicas e cutâneas também podem ocorrer. Quanto às formas mais graves e, felizmente raras, a SIM-P assume maior importância, podendo ter como alguns de seus desdobramentos a necessidade de suporte de terapia intensiva e o cuidado multiprofissional intensivo (Sociedade Brasileira de Pediatria, 2020; Fiocruz, 2020).

O espectro clínico da SIM-P é diversificado e pode incluir, além da febre persistente, agressão aos sistemas respiratório, gastrointestinal, hematológico, dermatológico e cardiovascular, sendo os indicativos de lesões cardíacas significativos para a caracterização da doença. A síndrome é referida na maioria dos estados brasileiros, com cada vez menor número deles sem registros da patologia, e é predominante em São Paulo, Minas Gerais e na Bahia, unidades federativas que também apresentam grande quantidade de infectados pelo novo coronavírus, aparente causa inicial para o desenvolvimento da SIM-P. Tal informação contribui para retificar que, apesar dos resultados divulgados pela SBP, anteriormente citados, o aumento de diagnósticos confirmados de Covid-19, mesmo que resultando em quadros menos debilitantes, provavelmente implica na elevação de notificações da síndrome (Ferreira et al., 2020; Ministério da Saúde, 2021).

Quando se investiga a cronologia da síndrome no Brasil, percebe-se um crescimento exponencial que pode ser observado através do seguimento dos Boletins Epidemiológicos emitidos pelo MS. Sob esse aspecto, no período entre agosto de 2020 e junho de 2021, verificou-se um aumento de casos e óbitos na ordem de 412\% e 364\%, respectivamente. As variações também são notórias quando são analisados meses subsequentes de 2021, como o intervalo entre abril e junho, em que se atestou uma elevação aproximada de $23 \%$ na quantidade de casos de SIM-P. Esse avanço da doença tem sido desacompanhado por outras nações, a exemplo do que ocorre nos Estados Unidos, país que detinha mais relatos de SIM-P do 
que o Brasil e contabilizava a metade dos falecimentos aqui verificados, e que, no momento, apresenta contínua desaceleração semanal na expansão dessa patologia (Ministério da Saúde, 2020; Ministério da Saúde, 2021).

$\mathrm{Na}$ tentativa de melhor explicar o contexto epidemiológico que propicia o desenvolvimento da síndrome pediátrica pós-Covid, tem-se tentado checar a existência de fatores de risco que favoreçam o surgimento desta condição em determinados pacientes. Apesar de estes não serem precisamente definidos, existem hipóteses sobre a suscetibilidade genética associada ao desenvolvimento da SIM-P, assim como evidenciado na DK, que sugerem que as mutações em genes que regulam a função de células de defesa permitam o desencadeamento de um intenso processo inflamatório, explicando o motivo de apenas uma minoria de crianças e adolescentes evoluírem com a síndrome, dada a infrequência dessas modificações no genoma humano. Dessa forma, há expectativas de que, caso a teoria seja, posteriormente, confirmada, intervenções médicas possam ser antecipadas em crianças com maiores chances de cursar com a SIM-P e, assim, reduzam a ocorrência de desfechos negativos associados a ela (Esposito et al., 2021).

Ainda sobre a possível existência de causas determinantes, a uniformidade encontrada na composição das amostras de diversas pesquisas que investigaram jovens com SIM-P levanta novos questionamentos sobre o tema. Apesar de não ser classificado como fator predisponente ao surgimento da síndrome, assim como as mutações genéticas, o sexo masculino representou a maior parcela acometida em repetidos estudos, como naqueles que foram concretizados no Chile, Brasil e Qatar, com distribuição que oscilou entre $52 \%$ e $71,4 \%$ da população avaliada, sem que haja explicações para esses resultados. Também, a alta incidência em pessoas negras, compondo cerca de 40\% dos casos de SIM-P nos trabalhos de Whittaker et al. (2020) e Payne et al. (2021), pôde ser confirmada em variadas produções científicas. Uma vez que não se sabe ao certo se tais dados indicam não somente uma coincidência, não há conclusões sobre a existência de grupos de risco, o que exige iguais cuidados preventivos por parte de todos os pacientes pediátricos.

Apesar de a síndrome inflamatória multissistêmica temporalmente associada à Covid-19 ser mais frequentemente relacionada às crianças e adolescentes, recentemente tem-se avaliado sua extensão para outras faixas etárias. Análise feita pelo CDC, que avaliou uma série de casos com manifestações similares àquelas vistas no público infanto-juvenil, destacou o reconhecimento de uma síndrome inflamatória multissistêmica em adultos (SIM-A) pós-Covid-19. Sua semelhança com a SIM-P é explicada pelo comprometimento cardiovascular acentuado, observado em 100\% da amostra populacional em questão, e por um acometimento respiratório ameno, opondo-se, no entanto, à insuficiência respiratória comumente descrita em pacientes hospitalizados com Covid-19 grave. Apesar de essa diferenciação contribuir para o efetivo diagnóstico da SIM-A, testes de reação em cadeia da polimerase (PCR) ou testes sorológicos para detecção de marcadores para a infecção pelo SARSCoV-2 são imprescindíveis para a confirmação da suspeita clínica (Morris et al., 2020).

Informações oficiais, em âmbito federal, sobre a evolução do estado de saúde dos pacientes acometidos pela SIM-P são de difícil acesso, restando, para efeito comparativo, boletins estaduais que discorrem sobre parâmetros como cura, internações em UTI e necessidade de suporte ventilatório. Nessa sequência, o estado de São Paulo, que registra elevados números de diagnósticos e mortes por SIM-P, apresentou percentuais aproximados de 73\%, 69\% e 26\%, respectivamente. Em estudo realizado nos Estados Unidos, com amostragem similar ao total de pacientes com SIM-P no estado paulista, mais de 90\% dos indivíduos diagnosticados com SIM-P se curaram e cerca de 79,5\% foram admitidos na UTI, sendo que, destes, $20 \%$ necessitaram de ventilação mecânica. Além dessas divergências numéricas, ressalta-se o fato de que, em São Paulo, relatos de cura se confundem com aqueles referentes às altas hospitalares, pois podem abranger situações que cursaram com sequelas pós-SIM-P, especialmente neurológicas, cardíacas e motoras, que compuseram, aproximadamente, 7,5\% dos dados classificados como "cura” (Centro de Vigilância Epidemiológica, 2021; Feldstein et al., 2020). 
Apesar dos registros de centros terciários, o pronto socorro pediátrico e os postos de saúde da rede de atenção primária consistem nas portas de entrada para crianças e adolescentes com suspeita de SIM-P, fato que traduz apreensões dos profissionais de saúde frente ao novo desafio, uma vez que a doença é relativamente recente. Na medida em que a SIM-P possui uma gama de sinais e sintomas comuns a diversas patologias e os achados laboratoriais se apresentam, na maioria das vezes, inespecíficos e abrangentes, o diagnóstico pode ser dificultado e levar a eventuais erros que possam prejudicar seu prognóstico (Hennon et al., 2020). Por esses motivos, ressaltam-se os papéis do médico generalista e/ou do pediatra quanto ao conhecimento desta condição para que a confirmação do caso aconteça em tempo hábil e ocorra a oferta adequada de cuidados a esses pacientes, tendo em vista a possibilidade de deterioração do quadro clínico nas horas e dias seguintes. Além disso, a capacitação e o poder resolutivo dos médicos da atenção básica evitam que encaminhamentos desnecessários sejam feitos aos hospitais, estratégia fundamental dado o contexto atual do país (Simon et al., 2020; Fiocruz, 2020).

Para além das questões que envolvem o diagnóstico precoce, a terapêutica a ser utilizada para a SIM-P ainda não possui consenso geral. Entretanto, a maioria das diretrizes nacionais e internacionais seguem as recomendações do Colégio Americano de Reumatologia, que preconiza o tratamento pautado pela tentativa de modular as reações hiperinflamatórias sistêmicas, adotando, para isso, uma combinação de medicamentos como imunoglobulina intravenosa (IgIV), glicocorticoides sistêmicos e aspirina (Pereira et al., 2020). De modo complementar, a SBP divulgou guias para o manejo desse quadro, alertando sobre a necessidade de internação dos pacientes com sintomatologia moderada ou grave, preferencialmente em hospitais com unidades de terapia intensiva pediátricas e com equipe multidisciplinar, o que, no atual cenário brasileiro, se torna inviável, em razão da alta taxa de ocupação hospitalar, podendo a espera por leitos levar à piora dos sintomas e, até mesmo, ao óbito (Sociedade Brasileira de Pediatria, 2020).

A iminência da volta às aulas presenciais, já aprovada em alguns estados, também é grande fonte de questionamentos no Brasil. O desenvolvimento, por parte de crianças e jovens, de sintomas e hábitos prejudiciais, em decorrência do isolamento social, como solidão, insônia, agitação e aumento excessivo do tempo de tela, corroboraram para que pais e responsáveis pressionassem autoridades públicas para que o retorno escolar fosse imediato. Tendo em vista os inúmeros relatos de desleixo dessa população quanto às medidas de preventivas em voga na pandemia da Covid-19, a exemplo do uso adequado de máscaras de proteção, da higienização das mãos e das restrições de contato interpessoal e de aglomerações, as exigências familiares representam um empecilho ao adequado controle da infecção no país. Diante desse histórico e do surgimento de repercussões como a SIM-P, a tomada de decisões que envolvam pacientes pediátricos, que antes não eram considerados fontes importantes de infecção e contágio, tem exigido maior cautela (De Alencar et al.,2020).

Nessa direção, frente à recente elevação do número de casos de SIM-P no Brasil e no mundo, desde a primeira confirmação diagnóstica, há uma maior vigilância genômica do novo coronavírus. A predominância global da mutação denominada D614G, que ocorre na proteína spike do novo coronavírus, associada à entrada do patógeno nas células humanas, é aventada como hipótese para justificar o desenvolvimento da síndrome inflamatória em determinados indivíduos, uma vez que outras alterações do genoma viral foram menos correlacionadas à ocorrência da SIM-P. Sendo esta também a mutação mais frequente no Brasil e havendo aumento de diagnósticos concomitantes à segunda onda da pandemia no país, período de maior diversidade de variantes do SARS-CoV-2, a continuidade do monitoramento genético pode permitir que se defina, posteriormente, se a síndrome se correlaciona a determinadas cepas ou a outras variantes, auxiliando na identificação de regiões maior predispostas para o encontro desses tipos virais (Parsons et al., 2021).

Ainda que se observe o avanço dessa síndrome no Brasil, ensaios clínicos que avaliem a implementação de vacinas contra a Covid-19 e que contemplem a faixa etária pediátrica permanecem em fase inicial. Atualmente, os imunizantes que receberam a autorização da Agência Nacional de Vigilância Sanitária (Anvisa) são os da Coronavac, AstraZeneca, Janssen e 
Pfizer, todos com permissão para uso apenas em maiores de 18 anos. No entanto, em junho de 2021, a última farmacêutica citada anunciou o avanço dos testes em crianças de 5 a 11 anos e iniciou o recrutamento da população entre 6 meses e 11 anos de idade para participação em estudos que visam a observação da eficácia e segurança da vacina contra a Covid-19, não sendo o Brasil, todavia, sede de nenhum dos testes a serem realizados. Quanto à CoronaVac, já aprovada para uso em pessoas de 3 a 17 anos, na China, e possuindo documentações que comprovam seu baixo perfil de efeitos adversos, esta aguarda liberação, pela Anvisa, para que possa ser aplicada em crianças maiores de 3 anos (Instituto Butantã, 2021; Nakara et al., 2020).

$\mathrm{Na}$ ausência de datas definitivas sobre a ampla distribuição dos imunizantes em todo o território nacional, e, frente à necessidade de conter os casos de Covid-19 e de SIM-P, cuidados preventivos continuam sendo imperiosos. Exemplos comportamentais e ensinamentos diários sobre medidas de prevenção, por parte dos pais e familiares, auxiliam, quando embasados em recomendações das autoridades em saúde, na obtenção da cooperação infantil para o retardo e, por vezes, impedimento da infecção pelo SARS-CoV-2, uma vez que devem englobar o uso correto de máscaras e a limpeza de mãos. Com relação aos adolescentes, a influência familiar também é preponderante, especialmente para o adequado cumprimento do isolamento social, ainda aconselhado no contexto pandêmico vigente. Dessa forma, e, mesmo após a autorização para uso dessas vacinas e o avanço da imunização, tais cuidados mantêm suas funções e recomendações, devendo ser exercitados, para o enfrentamento da pandemia, pelo tempo necessário, embora normas de higienização sejam atemporais (Esposito \& Principi, 2020; Fiocruz, 2020).

\section{Considerações Finais}

A SIM-P representa uma condição grave, recentemente associada à infecção pelo SARS-CoV-2. Desde que a notificação compulsória foi estabelecida no Brasil, os números de diagnósticos e mortes desencadeados por essa síndrome seguem uma curva crescente, principalmente no ano de 2021, período de maior avanço nos casos de Covid-19 no país. Em razão desta associação, a SIM-P reforça a necessidade de cautela por parte de crianças, adolescentes e de seus familiares quanto à adequada adoção de medidas preventivas recomendadas para a contenção do novo coronavírus. Ainda que se trate de tema recente, os números já notificados no país são suficientes para que pediatras e generalistas obtenham o devido conhecimento médico para o diagnóstico precoce desta síndrome, permitindo a redução dos desfechos negativos e seu melhor prognóstico. Tais dados ratificam, também, o papel fundamental da elaboração de novos estudos para melhor conhecimento dos aspectos contemplados por essa síndrome.

\section{Referências}

Alencar, M. F. R, Veloso, W. G., Bezerra, R. E. A., Gomes, L. A. \& Marcolino, A. B. L. (2021). O impacto da pandemia do covid-19 na saúde infanto-juvenil: um estudo transversal. Brazilian Journal of Health Review, 4, 1, 3483-3497.

Centro de Vigilância Epidemiológica. Vigilância Epidemiológica da Síndrome Inflamatória Multissistêmica Pediátrica (SIM-P), temporalmente associada à COVID-19 no Estado de São Paulo, Semanas Epidemiológicas 01/2020 a 06/2021. Boletim Epidemiológico, 1, 11, 1-6. https://docs.bvsalud.org/biblioref/2021/02/1148042/boletim11se6_simp.pdf.

Esposito, S. \& Principi, N. (2020). To mask or not to mask children to overcome COVID-19. European Journal of Pediatrics, 179, 8, 1267-1270.

Esposito, S. \& Principi, N. (2021). Multisystem Inflammatory Syndrome in Children Related to SARS-CoV-2. Pediatric Drugs, 23, 119-129.

Feldstein, L. R., Rose, L. B., Horwitz, S. M., Collins, J. P., Newhans, M. M., Son, M. B. F., Newburger, J. W., Kleinman, L. C., Heidemann, S. M., Martin, A. A., Singh, A. R. \& Li, S. (2020). Multisystem Inflammatory Syndrome in U.S. Children and Adolescents. The New England Journal of Medicine, 383, 334346. doi: 10.1056/NEJMoa2021680

Ferreira, B. W. R. C., Gusmão, A. B., de Leon, P. A. P., Ferreira, R. K. G. \& Macedo, C. L. (2021). Síndrome Inflamatória Multissistêmica Pediátrica (SIM-P) temporariamente associada à COVID-19: um levantamento das características clínicas e epidemiológicas. Research, Society and Development, 10, 3, e5710313020

Fiocruz (2021). Instituto Nacional de Saúde da Mulher, da Criança e do Adolescente Fernando Figueira. Covid-19 e saúde da criança e do adolescente, 1-70. 
Fontes, L. G. M., Saavedra, R. C., Carvalho, J. M. A., Broucke, V. R. B. V., Araújo, F. A. \& Menezes, G. A. (2021). Síndrome Inflamatória Multissistêmica Pediátrica (SIM-P) na Bahia, em 2020. Revista Baiana de Saúde Pública, 45(1), 46-61.

Godfred-Cato, S., Bryant, B., Leung, J., Oster, M. E., Conklin, L., Abrams, J., Roguski, K., Wallace, B., Prezzato, E., Koumans, E. H., Lee, E. H., Geevarughese, A., Lash, M. K., Reilly, K. H., Pulver, W. P., Thomas, D., Feder, K. A., Hsu, K. K., Plipat, N., Richardson, G., Reid, H., Lim, S., Schmitz, A., Pierce, T., Hrapcak, S., Datta, D., Morris, S. B., Clarke, K. \& Belay, E. (2020). COVID-19-Associated Multisystem Inflammatory Syndrome in Children United States, March-July 2020. Morbidity and Mortality Weekly Report, 69, 32, 1074-1080.

Instituto Butantã (2021). CoronaVac é segura e gera forte resposta imune em crianças e adolescentes, confirma estudo. https://butantan.gov.br/noticias/coronavac-e-segura-e-gera-forte-resposta-imune-em-criancas-e-adolescentes-confirma-estudo

Hasan, M. R., Al Zubaidi, K., Diab, K., Hejazi, Y., Bout-Tabaju, S., Al-Adba, B., Al Maslamani, E., Janahi, M., Roscoe, D., Lopez, A. P. \& Tang, P. (2021). COVID-19 related multisystem inflammatory syndrome in children (MIS-C): a case series from a tertiary care pediatric hospital in Qatar. BMC Pediatrics, 21, 267.

Hennon, T. R., Penque, M. D., Abdul-Aziz, R., Alibrahim, O. S., McGreevy, M. B., Prout, A. J., Schaefer, B. A., Ambrusko, S. J., Pastore, J. V., Turkovich, S. J., Gomez-Duarte, O. G., \& Hicar, M. D. (2020). COVID-19 associated Multisystem Inflammatory Syndrome in Children (MIS-C) guidelines; a Western New York approach. Progress in pediatric cardiology, 101232.

Lima-Setta, F., Magalhães-Barbosa, M. C., Rodrigues-Santos, G., Figueiredo, E., Jacques, M. L., Zeitel, R. S., Sapolnik, R., Borges, C., Lanziotti, V. S., Castro, R., Bellinat, A., Silva, T., Oliveira, F., Reis, B., Castro, N., Macedo, J., Scarlato, A., Riveiro, P. M., Mota, I., Lorenzo, V. B., de Lucena, N. M. L., Azevedo, Z. M. A., Cunha, A. J. L. A. \& Prata-Barbosa, A. (2021). Multisystem inflammatory syndrome in children (MIS-C) during SARS-CoV-2 pandemic in Brazil: a multicenter, prospective cohort study. Jornal de pediatria, 97, 3, 354-361.

Martins, G. M. V., Rangel, S. S., Eduardo, M. P., Rosário, I. R. A. S. \& Dias, E. C. C. A. (2020). Síndrome Inflamatória Multissistêmica Pediátrica Associada ao COVID-19. Revista Científica da FMC, 15, 3, 77-81.

Ministério da Saúde, M. S. (2021). Secretaria de Vigilância em Saúde. Boletim Epidemiológico, 52, 23. https://www.gov.br/saude/ptbr/media/pdf/2021/junho/21/boletim_epidemiologico_svs_23.pdf

Ministério da Saúde, M. S. (2021). Painel Coronavírus. Coronavírus Brasil. Recuperado de: https://covid.saude.gov.br/

Ministério da Saúde, M. S. (2021). Vacinômetro. https://www.gov.br/saude/pt-br/vacinacao

Morris, S. B., Schwartz, N. G., Patel, P., Abbo, L., Beauchamps, L., Balan, S., Lee E. H., Paneth-Pollak, R., Geevarughese, A., Lash, M. K., Dorsinville M. S., Ballen, V., Eiras, D. P., Newton-Cheh, C., Smith, E., Robinson, S., Stogsdill, P., Lim, S., Fox, S. E., Richardson, G., Hand, J., Oliver, N. T., Kofman, A., Bryant, B., Ende, Z., Datta, D., Belay, E., \& Godfred-Cato, S. (2020). Case series of multisystem inflammatory syndrome in adults associated with SARSCoV-2 infection - United Kingdom and United States, March-August 2020. Morbidity and Mortality Weekly Report, 69, 40, 1450.

Nakara, N. A., Blumberg, D. A., Herrera-Guerra, A., \& Lakshminrusimha, S. (2020). Multi-System Inflammatory Syndrome in Children (MIS-C) Following SARS-CoV-2 Infection: Review of Clinical Presentation, Hypothetical Pathogenesis, and Proposed Management. Children (Basel, Switzerland), 7, 7, 69.

Organização Mundial da Saúde (2020). Virtual press conference on COVID-19 - 11 march 2020. https://www.who.int/docs/defaultsource/coronaviruse/transcripts/who-audio-emergencies-coronavirus-press-conference-full-and-final-11mar2020.pdf

Parsons, E., Timlin, M., Starr, C., Fries, A., Wells, R., Studer, M. \& Sainato, R. (2021). Multisystem Inflammatory Syndrome in Children in February 2020 and Implications of Genomic Sequencing for SARS-CoV-2. Journal of the Pediatric Infectious Diseases Society, 10, 5, 695-697.

Passarinho, N. \& Barrucho, L. (2021). As crianças mortas por Covid no Brasil: "Meu filho poderia ter sobrevivido se tivesse sido testado". BBC News Brasil. https://www.bbc.com/portuguese/geral-56775977

Payne, A. B., Gilani, Z., Godfred-Cato, S., Belay, E. D., Feldstein, L. R., Patel, M. M., Randolph, A. G., Newhams, M., Thomas, D., Magleby, R., Hsu, K., Burns, M., Dufort, E., Maxted, A., Pietrowski, M., Longenberger, A., Bidol, S., Henderson, J., Sosa, L., Edmundson, A., Tobin-D'angelo, M., Edison, L., Heidemann, S., Singh, A. R., Giuliano, J. S., Kleinman, L. C., Tarquinio, K. M., Carrol, C. L., Hoots, B. E., Reed, C., Dahlgren, F. S., Oster, M. E., Pierce, T. J., Curns, A. T., Langley, G. E., Campbell, A. P., Balachandran, N., Murray, T. S., Burkholder, C., Brancard, T., Lifshitz, J., Leach, D., Charpie, I., Tice, C., Coffin, S. E., Perella, D., Jones, K., Marohn, K. L., Yager, P. H., Fernandes, N. D., Flori, H. R., Konicki, M. L., Walker, K. S., Di Pentima, M. C., Li, S., Horwitz, S. M., Gaur, S., Coffey, D. C., Harwayne-Gidansky, I., Hymes, S. R., Thomas, N. J., Ackerman, K. G. \& Cholette, J. M. (2021). Incidence of Multisystem Inflammatory Syndrome in Children Among US Persons Infected With SARS-CoV-2. JAMA Network Open, 4, 6, e2116420.

Pereira, M. F. B., Litvinov, N., Farhat, S. C. L., Eisencraft, A. P., Gibelli, M. A. B. C., Carvalho, W. B., Fernandes, V. R., Fink, T. T., Framil, J. V. S., Galleti, K. V., Fante, A. L., Fonseca, M. F. M., Watanabe, A., Paula, C. S. Y., Palandri, G. G., Leal, G. N., Diniz, M. F. R., Pinho, J. R. R. \& Silva, C. A. (2020). Severe clinical spectrum with high mortality in pediatric patients with COVID-19 and multisystem inflammatory syndrome. Clinics, 75.

Rowley A. H. (2020). Understanding SARS-CoV-2-related multisystem inflammatory syndrome in children. Nature reviews. Immunology, $20,8,453-4$.

Sáfadi, M. A. P (2020). As características intrigantes da COVID-19 em crianças e seu impacto na pandemia. Jornal de Pediatria, 96, 3, 265-268.

Sociedade Brasileira de Pediatria (2020). Síndrome inflamatória multissistêmica em crianças e adolescentes provavelmente associada à COVID-19: uma apresentação aguda, grave e potencialmente fatal. Departamento Científico de Infectologia. https://www.sbp.com.br/fileadmin/user_upload/22532dNA_Sindr_Inflamat_Multissistemica_associada_COVID19.pdf

Silva, J. R. A. (2020). Covid-19 em pediatria: um panorama entre incidência e mortalidade. Residência Pediátrica, 10, 3, 1-4.

Simon, H. J., Sakano, T., Rodrigues, R. M., Eisencraft, A. P., Carvalho, V., Schvartsman, C., \& Reis, A. (2021). Multisystem inflammatory syndrome associated with COVID-19 from the pediatric emergency physician's point of view. Jornal de Pediatria, 97, 2, 140-159. 
Research, Society and Development, v. 10, n. 9, e44710918154, 2021

(CC BY 4.0) | ISSN 2525-3409 | DOI: http://dx.doi.org/10.33448/rsd-v10i9.18154

Torres, J. P., Izquierdo, G., Acuña, M., Pavez, D., Reyes, F., Fritis, A., González, R., Rivacoba, C., Contardo, V. \& Tapia, L. I. (2020). Multisystem inflammatory syndrome in children (MIS-C): Report of the clinical and epidemiological characteristics of cases in Santiago de Chile during the SARS-CoV-2 pandemic. IJID: official publication of the International Society for Infectious Diseases, 100, 75-81.

Whittaker, E., Bamford, A., Kenny, J., Kaforou, M., Jones, C. E., Shah, P., Ramnarayan, P., Fraisse, A., Miller, O., Davies, P., Kucera, F., Brierley, J., McDougall, M., Carter, M., Tremoulet, A., Shimizu, C., Herberg, J., Burns, J. C., Lyall, H. \& Levin, M. (2020). Clinical Characteristics of 58 Children With a Pediatric Inflammatory Multisystem Syndrome Temporally Associated With SARS-CoV-2. JAMA, 324, 3, 259-269. 